\title{
Characterization of Generalized Uniform Distribution through Expectation
}

\author{
Milind B. Bhatt \\ Department of Statistics, Sardar Patel University, Vallabh Vidhyanagar, India \\ Email: bhattmilind b@yahoo.com
}

Received 29 April 2014; revised 25 May 2014; accepted 15 June 2014

Copyright (C) 2014 by author and Scientific Research Publishing Inc.

This work is licensed under the Creative Commons Attribution International License (CC BY).

http://creativecommons.org/licenses/by/4.0/

(c) $\underset{\mathrm{EY}}{\mathrm{B}}$ Open Access

\begin{abstract}
Normally the mass of a root has a uniform distribution but some have different uniform distributions named Generalized Uniform Distribution (GUD). The characterization result based on expectation of function of random variable has been obtained for generalized uniform distribution. Applications are given for illustrative purpose including a special case of uniform distribution.
\end{abstract}

\section{Keywords}

Characterization, Generalized Uniform Distribution (GUD)

\section{Introduction}

Normally the mass of a root has a uniform distribution. Plant develops into the reproductive phase of growth; a mat of smaller roots grows near the surface to a depth of approximately 1/6-th of maximum depth achieve (see G. Ooms and K. L. Moore [1]). Dixit [2] studied the problem of efficient estimation of parameters of a uniform distribution in the presence of outliers. He assumed that a set of random variables $X_{1}, X_{2}, \cdots, X_{n}$ represents the masses of roots where out of $n$-random variables some of these roots (say $k$ ) have different masses; therefore, those masses have different uniform distributions with unknown parameters and these $k$ observations are distributed with Generalize Uniform Distribution (GUD) with probability density function (pdf)

$$
f(x ; \theta)= \begin{cases}\frac{\alpha+1}{\theta^{\alpha+1}} x^{\alpha} ; & \alpha>-1, a \leq x<\theta \leq b, a \equiv 0 \\ 0 ; & \text { otherwise. }\end{cases}
$$

where $-\infty \leq a<b \leq \infty$ are known constants; $X^{\alpha}$ is positive absolutely continuous function and $(1 / \theta)^{\alpha+1}$ is everywhere differentiable function. Since derivative of $X^{\alpha+1}$ being positive, range is truncated by $\theta$ from right $a \equiv 0$.

Dixt [3] obtained Maximum Likelihood Estimator (MLE) and the Uniformly Minimum Variance (UMVU) 
estimator of reliability functional, $P[X>Y]$ in the same setup and showed that the UMVUE is better than MLE when one parameter of GUD is known, where as both parameters of the GUD are unknown, $P[X>Y]$ is estimated by using mixture estimate and is consistent.

In this paper the problem of characterization of GUD with pdf given in (1.1) has been studied and the characterization also holds for uniform distribution on interval $(a, \theta)$ when $\alpha=0$. Various approaches were used to characterize uniform distribution; few of them have used coefficient of correlation of smaller and the larger of a random sample of size two; Bartoszyn'ski [4], Terreel [5], Lopez-Bldzquez [6] as Kent [7], have used independence of sample mean and variance; Lin [8], Too [9], Arnold [10], Driscoll [11], Shimizu [12], and Abdelhamid [13] have used moment conditions, $n$-fold convolution modulo one and inequalities of Chernoff-type were also used (see Chow [14] and Sumrita [15]).

In contrast to all above brief research background and application of characterization of member of Pearson family, this research does not provide unified approach to characterized generalized uniform.

The aim of the present research note is to give a path breaking new characterization for generalized uniform distribution through expectation of function of random variable, $\phi(X)$ using identity and equality of expectation of function of random variable. Characterization theorem was derived in Section 2 with method for characterization as remark and Section 3 devoted to applications for illustrative purpose including special case of uniform distribution.

\section{Characterization}

Theorem 2.1. Let $X$ be a continuous random variable (rv) with distribution function $F(X)$ having pdf $f(x ; \theta)$. Assume that $F(X)$ is continuous on the interval $(a, b)$, where $-\infty \leq a<b \leq \infty$. Let $g(X)$ be a differentiable functions of $X$ on the interval $(a, b)$, where $-\infty \leq a<b \leq \infty$ and more over $g(X)$ be nonconstant. Then $f(x ; \theta)$ is the pdf of Generalize Uniform Distribution (GUD) defined in (1.1) if and only if

$$
E\left[g(X)+\left(\frac{X}{\alpha+1}\right) \frac{\mathrm{d}}{\mathrm{d} X} g(X)\right] .
$$

Proof: Given $f(x ; \theta)$ defined in (1.1), if $\phi(X)$ is such that $g(\theta)=E[\phi(X)]$ where $g(\theta)$ is differentiable function then

$$
g(\theta)=\int_{a}^{\theta} \phi(x) f(x ; \theta) \mathrm{d} x
$$

Differentiating with respect to $\theta$ on both sides of (2.2) and replacing $X$ for $\theta$ after simplification one gets

$$
\phi(X)=g(X)+\left(\frac{X}{\alpha+1}\right) \frac{\mathrm{d}}{\mathrm{d} X} g(X) .
$$

which establishes necessity of (2.1). Conversely given (2.1), let $k(x ; \theta)$ be the pdf of rv $X$ such that

$$
g(\theta)=\int_{a}^{\theta}\left[g(X)+\left(\frac{X}{\alpha+1}\right) \frac{\mathrm{d}}{\mathrm{d} X} g(X)\right] k(x ; \theta) \mathrm{d} x .
$$

Since $a=0$, the following identity holds

$$
g(\theta)=\left(\frac{\alpha+1}{\theta^{\alpha+1}}\right) \int_{a}^{\theta}\left[\frac{\mathrm{d}}{\mathrm{d} x}\left\{\left(\frac{x^{\alpha+1}}{\alpha+1}\right) g(X)\right\}\right] \mathrm{d} x .
$$

Differentiating $\left(\frac{x^{\alpha+1}}{\alpha+1}\right) g(x)$ with respect to $x$ and simplifying after tacking $\frac{\mathrm{d}}{\mathrm{d} x}\left(\frac{x^{\alpha+1}}{\alpha+1}\right)$ as one factor one gets (2.5) as

$$
g(\theta)=\int_{a}^{\theta}\left[g(x)+\left\{\frac{\frac{x^{\alpha+1}}{\alpha+1}}{\frac{\mathrm{d}}{\mathrm{d} x}\left(\frac{x^{\alpha+1}}{\alpha+1}\right)}\right\} \frac{\mathrm{d}}{\mathrm{d} X} g(X)\right]\left\{\left(\frac{\alpha+1}{\theta^{\alpha+1}}\right) \frac{\mathrm{d}}{\mathrm{d} x}\left(\frac{x^{\alpha+1}}{\alpha+1}\right)\right\} \mathrm{d} x .
$$


Substituting derivative of $\left(x^{\alpha+1} / \alpha+1\right)$ in (2.6) it reduces to

$$
g(\theta)=\int_{a}^{\theta} \phi(X)\left\{\left(\frac{\alpha+1}{\theta^{\alpha+1}}\right) x^{\alpha+1}\right\} \mathrm{d} x .
$$

where $\phi(X)$ is derived in (2.3) and by uniqueness theorem from (2.4) and (2.7)

$$
k(x ; \theta)=\frac{\alpha+1}{\theta^{\alpha+1}} x^{\alpha+1}
$$

Since $(1 / a)^{\alpha}$ is decreasing function for $-\infty \leq a<b \leq \infty$ and $a^{\alpha+1}=0$ is satisfy only when range of $X$ is truncated by $\theta$ from right and integrating (2.8) on the interval $(a, \theta)$ on both sides, one gets $k(x ; \theta)$ derived in (2.8) as

$$
k(x ; \theta)=\frac{\alpha+1}{\theta^{\alpha+1}} x^{\alpha} ; \alpha>-1, a \leq x<\theta \leq b, a \equiv 0 .
$$

and

$$
1=\int_{a}^{\theta} k(x ; \theta) \mathrm{d} x
$$

Hence $k(x ; \theta)$ derived in (2.9) reduces to $f(x ; \theta)$ defined in (1.1) which establishes sufficiency of (2.1).

Remark 2.1. Using $\phi(X)$ derived in (2.3), the $f(x ; \theta)$ given in (1.1) can be determined by

$$
M(X)=\frac{\frac{\mathrm{d}}{\mathrm{d} X} g(X)}{\phi(X)-g(X)} .
$$

and pdf is given by

$$
f(x ; \theta)=\frac{\frac{\mathrm{d}}{\mathrm{d} X} T(X)}{T(\theta)} .
$$

where $T(X)$ is increasing function for $-\infty \leq a<b \leq \infty$ with $T(a)=0$ such that it satisfies

$$
M(X)=\frac{\mathrm{d}}{\mathrm{d} X}[\log T(X)] .
$$

Remark 2.2. If $\alpha=0$ characterization theorem 2.1 also holds for uniform distribution with pdf

$$
f(x ; \theta)= \begin{cases}\frac{1}{\theta} ; & a \leq x<\theta \leq b, a \equiv 0 \\ 0 ; & \text { otherwise. }\end{cases}
$$

\section{Examples}

Using method describe in remark 2.1 Generalize Uniform Distribution (GUD) through expectation of non-constant function of random variable such as mean, $r^{\text {th }}$ raw moment, $\mathrm{e}^{\theta}, \mathrm{e}^{-\theta}, p^{\text {th }}$ quantile, distribution function, reliability function and hazard function is given to illustrate application and significant of unified approach of characterization result (2.1) of theorem 2.1.

Example 3.1. Characterization of Generalize Uniform Distribution (GUD) through hazard function

$$
g(\theta)=\frac{\left(\frac{\alpha+1}{\theta}\right)\left(\frac{t}{\theta}\right)^{\alpha}}{1-\left(\frac{t}{\theta}\right)^{\alpha+1}}
$$

therefore 


$$
g(X)=\frac{\left(\frac{\alpha+1}{X}\right)\left(\frac{t}{X}\right)^{\alpha}}{1-\left(\frac{t}{X}\right)^{\alpha+1}}
$$

From (2.3) one gets $\phi(X)$ as

$$
\phi(X)=g(X)+\left(\frac{X}{\alpha+1}\right) \frac{\mathrm{d}}{\mathrm{d} X} g(X)=-\frac{(\alpha+1) t\left(\frac{t}{X}\right)^{2 \alpha}}{\left[1-t\left(\frac{t}{X}\right)^{\alpha}\right]^{2}} .
$$

and using (2.10) of remark 2.1 the

$$
M(X)=\frac{\frac{\mathrm{d}}{\mathrm{d} X} g(X)}{\phi(X)-g(X)}=\frac{1+\alpha}{X} .
$$

By characterization method describe in remark 2.1, if

$$
\frac{\mathrm{d}}{\mathrm{d} X} \log \left[\frac{X^{a+1}}{a+1}\right]=M(X)
$$

then

$$
T(X)=\frac{X^{a+1}}{a+1}
$$

and substituting $T(X)$ as appeared in (2.12) for (2.11),

$$
f(x ; \theta)=\frac{\frac{\mathrm{d}}{\mathrm{d} x} T(x)}{T(\theta)}=\frac{\alpha+1}{\theta^{\alpha+1}} x^{\alpha} .
$$

is characterized.

Example 3.2. The characterization of $f(x ; \theta)$ defined in (2.1) through non constant function such as

$$
g_{i}(\theta)= \begin{cases}\frac{\alpha+1}{\alpha+2} \theta ; & \text { for } i=1, \text { mean, } \\ \frac{\alpha+1}{\alpha+r+1} \theta^{r} ; & \text { for } i=2, r^{\text {th }} \text { moment, } \\ \mathrm{e}^{\theta} ; & \text { for } i=3, \\ \mathrm{e}^{-\theta} ; & \text { for } i=4, \\ \theta p^{1 / \alpha+1} ; & \text { for } i=5, p^{\text {th }} \text { quantile, } \\ \frac{\left(\frac{t}{\theta}\right)^{\alpha+1} ;}{1-\left(\frac{t}{\theta}\right)^{\alpha+1} ;} ; & \text { for } i=6, \text { distribution function, } \\ \left(\frac{\alpha+1}{\theta^{\alpha+1}}\right)^{\alpha} & \text { for } i=8, \text { hazard function. } \\ 1-\left(\frac{t}{\theta}\right)^{\alpha+1} & \end{cases}
$$

and using 


$$
\left[\phi(X)-g_{i}(X)\right]= \begin{cases}\frac{X}{\alpha+2} ; & \text { for } i=1, \text { mean, } \\ \frac{r X^{r}}{\alpha+r+1} ; & \text { for } i=2, r^{\text {th }} \text { moment, } \\ \frac{X \mathrm{e}^{X}}{\alpha+1} ; & \text { for } i=3, \\ -\frac{X \mathrm{e}^{X}}{\alpha+1} ; & \text { for } i=4, \\ -\left(\frac{t}{X}\right)^{\alpha+1} ; & \text { for } i=5, p^{\text {th }} \text { quantile, } \\ \left(\frac{t}{X}\right)^{\alpha+1} ; & \text { for } i=6, \text { distribution function, } \\ \left.-\frac{(\alpha+1)\left(\frac{t}{X}\right)^{\alpha}}{X^{2}\left[\left(\frac{t}{X}\right)^{\alpha+1}-1\right.}\right]^{2} & \text { for } i=8, \text { hazard function. }\end{cases}
$$

and defining $M(X)$ given in (2.10) and substituting $T(X)$ as appeared in (2.12) for (2.11), $f(x ; \theta)$ is characterized.

Example 3.3. In context of remark 2.2 uniform distribution with pdf given in (2.13) characterized through $p^{\text {th }}$ quantile

$$
g(\theta)=Q_{p}(\theta)=\theta p
$$

therefore

$$
g(X)=X p .
$$

From (2.3) one gets $\phi(X)$ as

$$
\phi(X)=g(X)+X \frac{\mathrm{d}}{\mathrm{d} X} g(X)=2 X p
$$

and using (2.10) of remark 2.1 the

$$
M(X)=\frac{\frac{\mathrm{d}}{\mathrm{d} X} g(X)}{\phi(X)-g(X)}=\frac{1}{X} .
$$

By characterization method describe in remark 2.1, if

$$
\frac{\mathrm{d}}{\mathrm{d} X} \log [X]=\frac{1}{X}=M(X)
$$

then

$$
\begin{gathered}
T(X)=X \\
f(x ; \theta)=\frac{\frac{\mathrm{d}}{\mathrm{d} x} T(x)}{T(\theta)}=\frac{1}{\theta}
\end{gathered}
$$

Example 3.3. The pdf $f(x ; \theta)$ defined in (2.13) can be characterized through non-constant function such as 


$$
g_{i}(\theta)= \begin{cases}\frac{\theta}{2} ; & \text { for } i=1, \text { mean, } \\ \frac{\theta^{r}}{r+1} ; & \text { for } i=2, r^{\text {th }} \text { moment, } \\ \mathrm{e}^{\theta} ; & \text { for } i=3, \\ \frac{\theta p}{-\theta} ; & \text { for } i=4, \\ \frac{t}{\theta} ; & \text { for } i=6, p^{\text {th }} \text { quantile, distribution function, } \\ 1-\frac{t}{\theta} ; & \text { for } i=7, \text { reliability function, } \\ \frac{1}{\theta-t} ; & \text { for } i=8, \text { hazard function. }\end{cases}
$$

and using

$$
\left[\phi_{i}(X)-g_{i}(X)\right]= \begin{cases}\frac{X}{2} ; & \text { for } i=1, \text { mean, } \\ \frac{r X^{r}}{r+1} ; & \text { for } i=2, r^{\text {th }} \text { moment, } \\ X \mathrm{e}^{X} ; & \text { for } i=3, \\ -X \mathrm{e}^{X} ; & \text { for } i=4, \\ X p ; & \text { for } i=5, p^{\text {th }} \text { quantile, } \\ -\frac{t}{X} ; & \text { for } i=6, \text { distribution function, } \\ \frac{t}{X} ; & \text { for } i=7, \text { reliability function, } \\ -\frac{X}{(X-t)^{2}} ; & \text { for } i=8, \text { hazard function. }\end{cases}
$$

and defining $M(X)$ given in (2.10) and substituting $T(X)$ as appeared in (2.12) for (2.11), $f(x ; \theta)$ is characterized.

\section{Conclusion}

To characterize pdf defined in (1.1) one needs any arbitrary non-constant function of $X$ which should be differentiable and integrable only.

\section{Acknowledgements}

This work is supported by UGC Major Research Project No: F.No.42-39/2013 (SR), dated 12-3-2013.

\section{References}

[1] Ooms, G. and Moore, K.L. (1991) A Model Assay for Genetic and Environmental Changes in the Architecture of Intact Roots Systems of Plants Grown in Vitro. Plant Cell, Tissue and Organ Culture, 27, 129-139. http://dx.doi.org/10.1007/BF00041281

[2] Dixit, U.J., Ali, M. and Woo, J. (2003) Efficient Estimation of Parameters of a Uniform Distribution in the Presence of Outliers. Soochow Journal of Mathematics, 29, 363-369.

[3] Dixit, U.J. and Phal, K.D. (2009) Estimation of P (X Y) for the Uniform Distribution in the Presence of Outliers. Journal of Probability and Statistical Science, 7, 11-18.

[4] Bartoszynski, R. (1980) Personal Communication. 
[5] Terrel, G.R. (1983) A Characterization of Rectangular Distributions. The Annals of Probability, 11, 823-826. http://dx.doi.org/10.1214/aop/1176993533

[6] Lopez-Bldzquez, F. and Salamanca Milño, B. (1999) On Terrers Characterization of Uniform Distribution. Statistical Papers, 40, 335-342. http://dx.doi.org/10.1007/BF02929879

[7] Kent, J.T., Mardia, K.V. and Rao, J.S. (1979) Characterization of the Uniform Distribution on the Circle. The Annals of Statistics, 7, 882-889. http://dx.doi.org/10.1214/aos/1176344737

[8] Lin, G.D. (1988) Characterizations of Uniform Distributions and of Exponential Distributions. Sankhyā A, 50, 64-69.

[9] Too, Y.H. and Lin, G.D. (1989) Characterizations of Uniform and Exponential Distributions. Statistics \& Probability Letters, 7, 357-359. http://dx.doi.org/10.1016/0167-7152(89)90051-5

[10] Arnold, B.C. and Meeden, G. (1976) A Characterization of the Uniform Distribution Based on Summation Modulo One, with Application to Fractional Backlogs. Australian Journal of Statistics, 18, 173-175. http://dx.doi.org/10.1111/j.1467-842X.1976.tb01294.x

[11] Driscoll, M.F. (1978) On Pairwise and Mutual Independence: Characterizations of Rectangular Distributions. Journal of the American Statistical Association, 73, 432-433. http://dx.doi.org/10.1080/01621459.1978.10481596

[12] Shimizu, R. and Huang, J.S. (1983) On a Characteristic Property of the Uniform Distribution. Annals of the Institute of Statistical Mathematics, 35, 91-94. http://dx.doi.org/10.1007/BF02480966

[13] Abdelhamid, S.N. (1985) On a Characterization of Rectangular Distributions. Statistics and Probability Letters, 3, 235238. http://dx.doi.org/10.1007/BF02480966

[14] Chow, Y. and Huang, S.Y. (1999) A Characterization of the Uniform Distribution via Moments of N-Fold Convolution modulo One. The Indian Journal of Statistics, 61, 148-151.

[15] Sumitra, P. and Subir, B. (1990) Characterization of Uniform Distributions by Inequalities of Chernoff-Type. The Indian Journal of Statistics, 52, 376-382. 
Scientific Research Publishing (SCIRP) is one of the largest Open Access journal publishers. It is currently publishing more than 200 open access, online, peer-reviewed journals covering a wide range of academic disciplines. SCIRP serves the worldwide academic communities and contributes to the progress and application of science with its publication.

Other selected journals from SCIRP are listed as below. Submit your manuscript to us via either submit@scirp.org or Online Submission Portal.
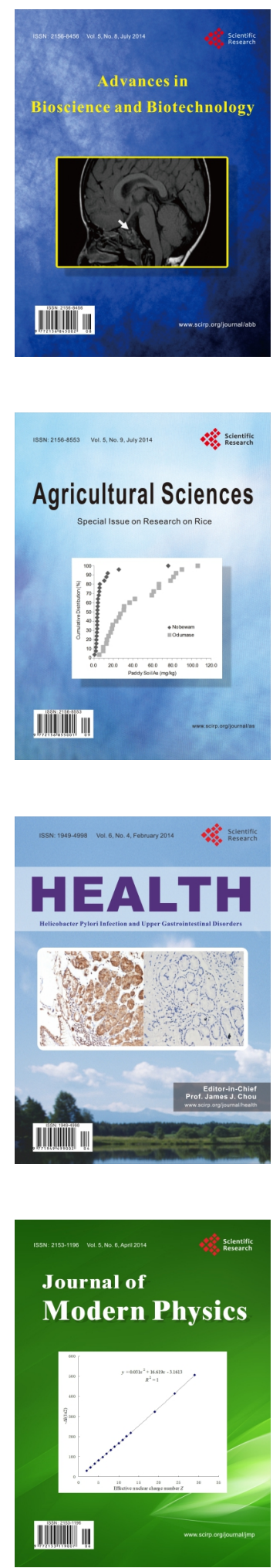
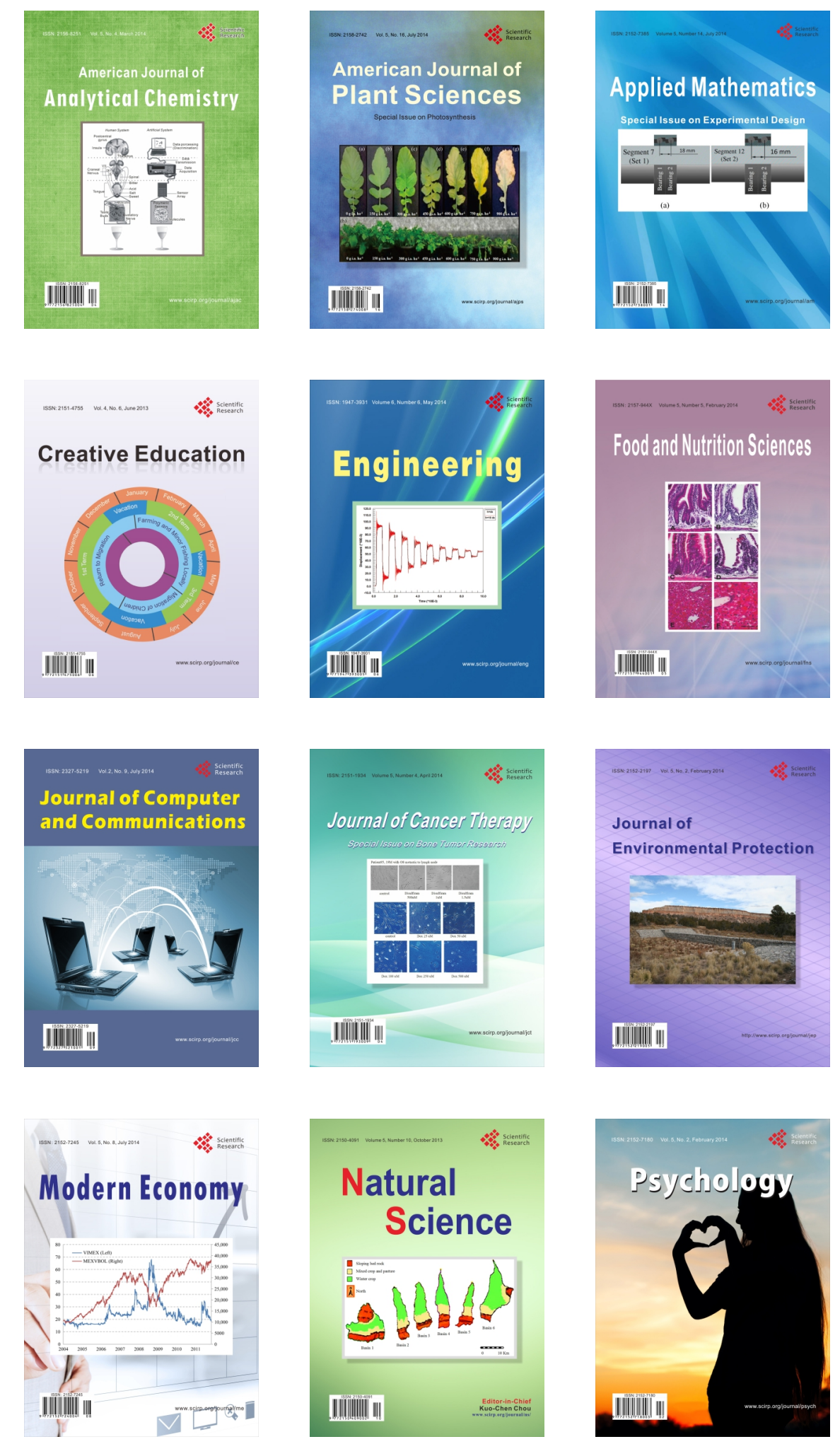